\title{
Sexualidad, diseminación y lectura
}

\author{
Raúl Quesada \\ La "doctrina" de un pensador es lo no dicho en su decir \\ y a lo que el hombre queda expuesto con el fin de \\ emplearse en ello. \\ Heidegger
}

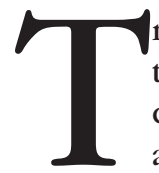

ratar de establecer lo que dice un filósofo cuando reflexiona sobre un tema es un ejercicio típicamente académico; tratar de entender por qué alguien evita hablar de algo, o guarda silencio sobre ello, es una actividad que paradigmáticamente, aunque no en forma exclusiva, pertenece a la psicología o al psicoanálisis; pero tratar de entender por qué un filósofo ha evitado o silenciado ciertos temas es, básicamente, un ejercicio de comprensión de su pensamiento y su escritura, y es reconocer de una manera explícita y con consecuencias interpretativas, que el silencio puede también ser parte constitutiva de lo que se dice y, en esa medida, de lo que se lee. De esta manera, podríamos abrir un signo de interrogación ante textos cuya lectura parece estar muy cerca de ciertos temas que se podrían considerar afines, o cercanos, o pertinentes, pero que literalmente nunca son tocados o mencionados por su autor, ¿qué derecho tendríamos entonces a acercarlos, a ponerlos en contacto? Por otro lado, ¿no es esto lo que hace toda lectura, relacionar un texto con otros textos, con otras lecturas? ¿Podríamos estar en la situación de buscarle tres pies al gato sabiendo que tiene..., cuántos? ¿De cuántos hilos está tejido un texto, cuál es el principal, qué se dice entre lo que se menciona y lo que se calla? Éstas son preguntas que cualquier lectura atenta suele propiciar y se vuelven pertinentes y hasta urgentes al leer un texto como "Geschlecht: différence sexuelle, différence ontologique", de Jacques Derrida; son pertinentes porque en ese texto, Derrida intenta una lectura de Heidegger alentada y, casi podríamos decir, si no fuera por la viva presencia de la noción de logocentrismo, centrada en su silencio acerca de la sexualidad; ¿se trata entonces de una forma de lectura que evita por principio, y desde el principio, la tentación de un logos dominante? Por otro lado, ¿cómo centrar o enfocar una lectura en un silencio que, por definición, es inaudible?

Tal vez podríamos empezar notando varios caminos o hilos que conducen hacia ese silencio, el primero es el de un núcleo privilegiado de $E l$ ser y el 
tiempo, el "ser ahí", el Dasein, ese ser, temporal, arrojado en el mundo, que es capaz de formular la pregunta que interroga por el ser; ese ser, que de acuerdo con Heidegger "somos en cada caso nosotros mismos", ese ser privilegiado, podemos nosotros preguntar, ¿está marcado por la sexualidad? Y, si lo está, ¿deja huella esa marca en su reflexión, ya sea ésta llanamente óntica o heideggerianamente ontológica? En otras palabras, ¿marca la sexualidad de alguna manera significativa el pensamiento del ser ahí? Una primera respuesta es negativa y suele ir acompañada de un jalón de orejas que despierte o avive nuestro seso filosófico; si leemos con un poco de cuidado el libro de Heidegger notaremos, en el parágrafo 10, el anunciado y preciso deslinde de la analítica del "ser ahí" con respecto a la antropología, la psicología y la biología. Siguiendo el espíritu del pensamiento heideggeriano, tal vez podríamos acentuar ese deslinde y extenderlo a las reflexiones que Derrida llama "filosofías de la vida", de esta manera debería quedar claro que la analítica del ser ahí no puede ser un trampolín para consideraciones que desbordan el ámbito de lo propiamente ontológico, el ámbito de lo fundamental. Por otro lado, cuando nuestra atención se enfoca en el Dasein, en ese ser que se pregunta por el ser y está, simplemente, ahí, notamos que, en consecuencia, su estructura fundamental es la de "ser en el mundo" y dicha estructura implica las de "ser con" y "ser sí mismo"; la pregunta entonces se hace patente: ¿cómo podemos estar en el mundo sin la marca de la sexualidad? Si, como insiste Heidegger, el ser ahí es el ente que "somos en cada caso nosotros mismos", entonces ¿cómo evadir la pregunta por la sexualidad y cómo hacer caso omiso del silencio heideggeriano con respecto a ella?

Un primer paso para mostrar la poca pertinencia de la pregunta por la sexualidad es señalar que su plausibilidad descansa en la cercanía entre el ser ahí y el existente, pero esta cercanía, se señala, no debe llevarnos a confundirlos, ya que esta confusión -antecedente notorio de ciertas reflexiones existencialistas-indicaría que no nos hemos percatado que la existencia misma tiene su fuente originaria (Urquell) en el Dasein, un ser, básicamente, neutro. Y, sin embargo, es precisamente esta neutralidad la que le servirá a Derrida como el hilo conductor o, con mayor precisión, deconstructor, que lo llevará a destejer el texto heideggeriano para examinar qué tan ausente está en ese texto, y en el Dasein mismo, la sexualidad. El punto de partida es un curso de verano que impartió Heidegger en 1928, acerca de Leibniz, inmediatamente después de la publicación de El ser y el tiempo, en la Universidad de Marburgo/ Lahn; ahí Heidegger enfatiza la diferencia entre el problema de la trascendencia y el problema al que dedica su libro. Ahí también, nos dice Derrida, Heidegger explica por qué la analítica del Dasein sólo puede presentarse bajo la perspectiva de una ontología fundamental: "Es porque no se trata allí ni de una 'antropología', ni de una 'ética'. Una analítica tal es solamente 'preparatoria' 
y la 'metafísica del Dasein' no está todavía 'en el centro' de la empresa, lo que hace claramente pensar que está, sin embargo, en su programa". ${ }^{1}$ Aquí, y no obstante la importancia de señalarlo, lo más notable no es tanto que una metafísica del Dasein pudiera formar parte de los afanes de Heidegger, sino el hecho de que el carácter neutral del Dasein esté marcado, en ese comentario, por una referencia a la diferencia sexual. En el curso de Marburgo, hace hincapié Derrida, Heidegger señala la neutralidad del Dasein, relacionándola abiertamente con la diferencia sexual y enfatizando su positividad originaria:

Esta neutralidad significa también que el Dasein no es ninguno de los dos sexos. Pero esta a-sexualidad (Geschlechtlosigkeit) no es la indiferencia de una nulidad vacía, la negatividad anuladora de una nada óntica indiferente. En su neutralidad, el Dasein no es el indiferente "ninguno y cada uno" (Niemand und Jeder), sino positividad originaria y el poderío del ser o de la esencia, Mächtigkeit des Wesens. ${ }^{2}$

De hecho, es precisamente esta neutralidad la que desplaza en el texto heideggeriano al sustantivo "hombre" para dar lugar al término neutro $\mathrm{Da}$ sein; esta elección la recuerda claramente Heidegger en el curso: "Para el ente que constituye el tema de esta analítica, no se ha elegido el título 'hombre' (Mensch), sino el título neutro 'das Dasein'". ${ }^{3}$ Pero si el o lo Dasein, y su correspondiente neutralidad, desplazan efectivamente al hombre y sus singularidades: ¿por qué, entonces, recalcar que tal neutralidad apunta en forma específica a la diferencia sexual?, y, sobre todo, ¿por qué señalar con tanto cuidado que la sexualidad excluida no se va a convertir o no es equivalente a una a-sexualidad (Geschlechtslosigkeit) indiferente, vacía, negativa, nula y anuladora? La secuencia misma de los adjetivos parece apuntar a la existencia de otra sexualidad o, al menos, de otra a-sexualidad, más acorde con el aura afirmativa y positiva que le es propia al Dasein: "positividad originaria", "fuerza del ser o de la esencia", "Mächtigkeit des Wesens".

Empecemos con el desplazamiento de Mensch (hombre); Derrida nos hace notar que si el ser ahí no equivale a "hombre", entonces es obvio que

${ }^{1}$ Jacques Derrida, "Geschlecht: différence sexuelle, différence ontologique", en Psyché: Inventions de l'autre. París, Galilée, 1987, p. 398. [Michel Haar, ed., Heidegger. París, L'Herne, 1983.]

${ }^{2}$ J. Derrida, "Choreographies", en Points... Interviews, 1974-1994. Ed. de Elizabeth Weber y trad. de Peggy Kamuf et al. Stanford, Universidad de Stanford, 1995, p. 104. [Entrevista con Christie V. McDonald, originalmente publicada en Diacritics 12, núm. 2, 1982.]

${ }^{3}$ J. Derrida, "Geschlecht: différence sexuelle, différence ontologique", en op. cit., p. 399. 
la distinción hombre/mujer no viene al caso, pero si esto es así, si es patente que el género no es un tema ontológicamente pertinente, ¿por qué molestarse en recordarlo?, ¿no basta haber señalado que la analítica del Dasein no es una empresa ni ética ni antropológica?, ¿o se trata acaso de una invitación indirecta a pensar que la diferencia sexual no puede confinarse con tal inmediatez a los ámbitos de una reflexión ética o antropológica? Empezando a trenzar los hilos de su argumentación dice Derrida:

La insistencia precautoria de Heidegger permite en todo caso pensar que las cosas no son tan obvias. Una vez que se ha neutralizado la antropología (fundamental o no) y mostrado que ésta no puede comprometer la cuestión del ser o estar allí comprometida en cuanto tal, una vez que se nota que el Dasein no se reduce ni al ser humano, ni al yo ( $m o i)$, ni a la conciencia ni al inconsciente, ni al sujeto ni al individuo, ni siquiera al animal racional, se podría creer que la cuestión de la diferencia sexual no tiene ninguna posibilidad de ser conmensurada con la cuestión del sentido del ser o de la diferencia ontológica y que aun su concedida ausencia no merece ningún trato privilegiado. ${ }^{4}$

Y sin embargo, afirma Derrida, lo que sucede es precisamente todo lo contrario ("c'est incontestablement le contraire qui se passe") y la razón principal para pensar de esta manera es advertir que tanto la neutralidad como la a-sexualidad (Geschlechtslosigkeit) tienen resonancias negativas que apuntan hacia la indiferencia y que claramente contrastan con el tono de la empresa heideggeriana y con la positividad fundamental del Dasein mismo. Si leemos con cuidado podremos notar, como lo hace Derrida, que los predicados negativos acaban resaltando nada menos que el poderío (Mächtigkeit) y la positividad originaria (ursprüngliche Positivität) que parecen consustanciales al Dasein. De esta manera se podría argüir que ni la neutralidad ni la a-sexualidad misma son suficientes para declarar que la sexualidad brilla por su ausencia en el proyecto de El ser y el tiempo. La aparente paradoja que conlleva esa conclusión se ve disminuida cuando, siguiendo a Derrida, circunscribimos la a-sexualidad del Dasein a la dualidad sexual y notamos que la negatividad ontológica asociada con una distinción genérica no se extiende, ipso facto, a la sexualidad misma. Esto básicamente quiere decir que la neutralidad asexual no desexualiza de por sí; muy por el contrario, si enfocamos la negatividad ontológica en la dualidad sexual entonces podría hacerse manifiesta una sexualidad sin resabios antropológicos, una sexualidad que más que ser opacada o silenciada por la neutralidad, la tomará como referencia de una

${ }^{4}$ Ibid., p. 401. 
distancia. Dice Derrida: "No habrá Geschlechtslosigkeit sino en relación con el 'dos'; la a-sexualidad no se determinará como tal sino en la medida en que por sexualidad se entienda inmediatamente binariedad o división sexual". 5 Es por esto, cree Derrida, que Heidegger puede afirmar que la a-sexualidad no es "la indiferencia de la nulidad vacía" (die Indifferenz des leeren Nichtigen), la débil negatividad de una nada óntica indiferente; estos señalamientos, por otro lado, podrían apuntar hacia la idea de que si bien el Dasein no pertenece a ninguno de los dos sexos, esto no quiere decir que no esté marcado por la sexualidad, que esté, como dice Derrida, privado o despojado de sexo (privé de sexe). Se abre así la puerta para empezar a pensar la sexualidad de otra manera, para pensarla sin la dualidad que suele envolverla y empezar a concebir lo que Derrida llama una "sexualidad pre-diferencial" o "pre-dual", una sexualidad "más originaria que la díada" (plus originaire que la dyade) y que podría ser relacionada con la positividad y el poderío a los que hace tan clara referencia Heidegger. Dice Derrida:

A partir de esta sexualidad más originaria que la díada [dyade], uno podría estar tentado a pensar en su fuente una "positividad" y un "poderío" que Heidegger tiene el cuidado de no llamar "sexuales", sin duda por temor a reintroducir la lógica binaria que la antropología y la metafísica siempre asignan al concepto de sexualidad. Sin embargo, ciertamente se trataría de la fuente positiva y poderosa de toda "sexualidad" posible. La Geschlechtslosigkeit no sería más negativa que la aletheia. ${ }^{6}$

Ahora, si la sexualidad originaria se apodera de la "positividad", entonces ocurre un desplazamiento, que Derrida califica de "extraño y muy necesario", por el cual será la división sexual misma la que lleva a la negatividad; de esta manera se puede pensar la neutralización como un efecto de esa negatividad y, a la vez, como una manera de ocultarla y eludirla para hacer patente una "positividad originaria". Por lo tanto, "Lejos de constituir una positividad que la neutralidad a-sexual del Dasein vendría a anular, la binariedad sexual sería ella misma la responsable -o, más bien, pertenecería a una determinación ella misma responsable- de esta negativización". ${ }^{7}$ De esta manera, Derrida podrá concluir que, a pesar de las apariencias, la a-sexualidad y la neutralidad que en el análisis heideggeriano habían sido separadas de la sexualidad binaria, ni se le oponen ni, de hecho, son tan distantes de ella. La lectura empieza entonces a mostrar sus retorcimientos y el mismo Derrida se pregunta si su interpre-

\footnotetext{
${ }^{5}$ Ibid., p. 402.

${ }^{6}$ Idem.

${ }^{7}$ Ibid., pp. 402-403.
} 
tación no se podría considerar demasiado violenta; según ella la asexualidad y la neutralidad que, de acuerdo con la analítica del Dasein, deberían, casi por definición, ser características que lo distanciaran e independizaran de la impronta sexual binaria, no logran esta independencia y se encuentran, de cierta manera, dentro del dominio de esa diferencia sexual binaria a la que, precisamente, parecían oponerse.

La interpretación podría considerarse violenta no sólo por el desplazamiento argumentativo que sugiere, sino porque carece de una deseable continuidad en el texto del Curso; como el propio Derrida señala, en los siguientes sub-parágrafos $(3,4,5)$ de ese texto, Heidegger, aun cuando hable de la neutralidad, la posibilidad y el poderío original, lo hará sin hacer referencia explícita a la diferencia sexual; de hecho, insiste Derrida, nunca usará el predicado "sexual" en relación con la palabra "poderío" (puissance), aunque esta restricción pudiera deberse a la precaución, muy pertinente, de evitar las asociaciones antropológicas y metafísicas de ese predicado. Por otro lado, en esas líneas, nos hace notar, se borrarán todos los signos de negatividad atribuibles a la neutralidad; este gesto es importante porque permite iluminar, sin la sombra de una neutralidad asociada con la binariedad, el camino hacia el poderío del origen, ese origen que porta "la posibilidad interna de la humanidad en su factualidad concreta [factualité concrète]". De aquí, como ya se mencionó, que no se deba confundir al Dasein con el existente, ya que este último depende del primero en la medida en la que tiene en él, en el $D a$ sein, su "fuente originaria" y su "posibilidad interna". De aquí también que los señalamientos de Derrida se formulen de una manera que los distancia de los ámbitos discursivos, típicamente binarios, que suelen acoger la reflexión sobre la sexualidad; sin embargo, tal vez sea precisamente esta distancia la que permita empezar a entender mejor los límites y limitaciones de la reflexión binaria sobre la sexualidad. Dice Derrida:

El Dasein no existe, desde luego, sino en su concreción factual, pero esta existencia misma tiene su fuente originaria (Urquell) y su posibilidad interna en el Dasein en tanto que neutro. La analítica de este origen no trata del existente mismo. Justamente porque las precede, esa analítica no puede confundirse con una filosofía de la existencia, con una sabiduría (que no se podría establecer sino dentro de la "estructura de la metafísica"), una profecía o una prédica que enseña una "visión del mundo". No es por lo tanto, de ninguna manera, una "filosofía de la vida". Esto quiere decir que un discurso acerca de la sexualidad que fuera de este orden (sabiduría, saber, metafísica, filosofía de la vida, o de la existencia) carecería de todas las exigencias de una analítica del Dasein, en su neutralidad misma. Ahora bien, ¿se ha presentado alguna 
vez un discurso acerca de la sexualidad que no pertenezca a uno de estos registros? ${ }^{8}$

Se trata entonces de pensar la sexualidad de otra manera, de una manera distinta de la que la casa de por vida con la diferencia sexual binaria, pero, sobre todo, de una manera que nos permita cuestionar la idea de que, en su neutralidad, el Dasein no está marcado por la sexualidad; esta posibilidad indicaría que el notorio silencio de Heidegger con respecto a esa marca, también puede ser leído de otra forma, de una forma que relaciona este silencio más con su precaución de no contaminar la analítica del ser ahí con reflexiones de otro orden (antropológico, psicológico, biológico o existencial), que con una decisión de privar al Dasein de un potencial sexual. Por otro lado, la posibilidad misma de que la neutralidad del Dasein no conlleve una a-sexualidad generalizada, sino sólo con referencia a su manifestación diádica, permitiría justificar cierta insistencia en otros asomos de sexualidad en la reflexión heideggeriana. Derrida hace referencia a otro texto de Heidegger, del mismo año que el curso de Marburgo, en este texto: Vom Wesen des Grundes se repite el acto de enfatizar la neutralidad en relación con la sexualidad y se vuelve a usar o, mejor dicho, puesto que se usarán comillas, se vuelve a mencionar la palabra Geschlechtlichkeit. Esta mención es importante por varias razones, en primer lugar, porque aparece en un contexto filosófico fundamental: el ser mismo del Dasein, su Selbsheit, su ipséité, su mismidad, en segundo lugar, porque dicha mención va marcada con cierta prioridad (erst recht) y, en tercer lugar, porque le permite a Derrida insistir en la posibilidad de acercar la sexualidad a las cuestiones ontológicas. En español esta cercanía, podríamos mencionar de paso, no se esconde: según el Diccionario Enciclopédico Espasa, ipsismo, una plausible traducción de ipséité, es sinónimo de masturbación. Claro está que, desde un punto de vista heideggeriano, este acercamiento, no por lingüístico menos escandaloso, está precisa y efectivamente contrarrestado por la neutralidad de la Selbsheit del Dasein; sin embargo, este ipsismo tiene sus bemoles. El Dasein, nos recuerda Derrida, existe por designio propio (dessein-de-soi), pero esto no quiere decir, como ya lo señalaba Heidegger, ni el para-sí de la conciencia, ni el egoísmo, ni el solipsismo, ya que la diferencia misma entre el "ser-yo" y el "ser-tú" (Ich-sein/Dusein) proviene de la Selbsheit. Consecuentemente, la Selbsheit (l'ipséité), será también neutral con respecto al ser-yo y al ser-tú, y con mayor razón, o en primer lugar, insistirá Heidegger, con respecto a la sexualidad; sin embargo, es precisamente ese énfasis el que usará Derrida para perfilar la idea de que la Selbsheit misma podría estar marcada por la sexualidad.

${ }^{8}$ Ibid., pp. 403-404. 
La reflexión de Derrida es la siguiente; en primer lugar, el énfasis aparece cuando se señala que, al estar siempre presupuesto, el ipsismo (l'ipséité) "es pues también 'neutro' con respecto del ser-yo y del ser-tú, 'y, con mayor razón, con respecto de la «sexualidad»' (und erst recht etwa gegen die 'Geschlechtlichkeit' neutral)". 9 La fuerza lógica de esta afirmación, marcada con un cierto a fortiori, depende, para Derrida, de que la "sexualidad" (entre comillas) "sea el predicado asegurado [assuré] de todo lo que se hace posible por o a partir del ipsismo", pero que, en tanto que "sexualidad", no puede pertenecer a la estructura del ipsismo, pues éste, recordemos, todavía no estará determinado como un ser humano o un sujeto consciente. Y, sin embargo, como en el caso del Dasein, si el razonamiento es tan claro, ¿por qué subrayar nuevamente la neutralidad con respecto a la sexualidad?, ¿indicará este énfasis, esta primacía (erst recht), la sombra de una sospecha, la sospecha de que la Selbsheit pudiera estar marcada por la sexualidad y que ésta, la sexualidad, pudiera invadir el ámbito de sus estructuras ontológicas? O, para asumir de una buena vez la violencia de la interpretación derridiana: y si el $D a$ del Dasein fuera ya sexual? Lo notorio de esta lectura es su tentadora invitación a considerarla no como una transgresión textual, sino como una exteriorización de la violencia escondida en el texto: la violencia de la neutralización. Dice Derrida:

Pero si Heidegger insiste y subraya ("con mayor razón"), es que todavía persiste una sospecha: ¿y si la "sexualidad” ya marcara la Selbsheit más originaria, si fuera una estructura ontológica del ipsismo, si el $D a$ del Dasein fuera ya "sexual"?, ¿y si la diferencia sexual estuviera ya marcada en la apertura a la cuestión del sentido del ser y a la diferencia ontológica?, ¿y si, puesto que no va de suyo, la neutralización fuera una operación violenta? El "con mayor razón" podría esconder una razón menor. ${ }^{10}$

A partir de esta incitación a cuestionar la neutralidad de la Selbsheit, se entreabre otra puerta a una posible dislocación del texto heideggeriano, relacionada también con la posibilidad de una lectura de la palabra Geschlecht que no estuviera limitada por la binariedad. A partir de esa lectura se podría pensar que otro "sexo", o, mejor dicho, otro Geschlecht pudiera marcar o inscribirse en el ipsismo y, consecuentemente, alterar "el orden de todas las derivaciones", notoriamente "aquella de una Selbsheit más originaria y que hiciera posible la emergencia del ego y del tú". ${ }^{11}$ Aquí las cosas se empiezan a

\footnotetext{
${ }^{9}$ Ibid., p. 404.

${ }^{10}$ Ibid., pp. 404-405.

${ }^{11}$ Ibid., p. 405.
} 
complicar más, ya que la dependencia del "ser-yo" y del "ser-tú" de un ipsismo neutral, apuntará hacia la distinción, bastante compleja y conflictiva, entre los análisis puramente ontológicos y neutrales del Dasein y los análisis que lo consideran "en el hombre" o, podríamos decir, encarnado. Derrida señala, por ejemplo, que la neutralidad del Dasein, que conlleva un cierto aislamiento, no implica una "soledad factual y existencial". Así, el problema que se empieza a perfilar con mayor nitidez es el de las relaciones entre el cuerpo y el Dasein; para tejer estas relaciones, Derrida no dejará de usar hilos específicamente heideggerianos, pero, no por ello, neutros y sin color, ya que al reconocerlos no podremos evitar los efectos de resonancia e irisación característicos de sus lecturas. Estos efectos son notorios en el siguiente párrafo:

El Dasein en general esconde, abriga en sí la posibilidad interna de una dispersión o de una diseminación factual (faktische Zerstreuung) en el cuerpo propio (Leiblichkeit) y, "por eso, en la sexualidad" (und damit in die Geschlechtlichkeit). Todo cuerpo propio está sexuado y no hay Dasein sin cuerpo propio. Pero el encadenamiento propuesto por Heidegger parece muy claro: la multiplicidad dispersante no se debe, en primer lugar, a la sexualidad del cuerpo propio; es el cuerpo propio mismo, la carne, la Leiblichkeit, la que arrastra originariamente al Dasein a la dispersión y, con ello [par suite], a la diferencia sexual. Este "con ello" (damit) es insistente en el intervalo de unas cuantas líneas, como si el Dasein debiera tener o ser a priori (como su "posibilidad interior") un cuerpo que se encuentra sexuado y afectado por la división sexual. ${ }^{12}$

La dispersión, la diseminación, la disociación, no son características contingentes del Dasein que puedan ser explicadas en términos de un accidente, de una caída, de un destronamiento, todo lo contrario, son inherentes a él en la medida que son manifestaciones de una estructura originaria; en consecuencia, esta estructura dispersante marcará y destinará tanto al Dasein como al cuerpo que inevitablemente lo encarna ("no hay Dasein sin cuerpo propio") y a la sexualidad inherente (puesto que "todo cuerpo propio está sexuado") hacia la multiplicidad, el desligamiento y la disociación. En su facticidad corporal el Dasein es "separado, sumido en la dispersión y la fragmentación (zersplittert) y, por lo mismo (ineins damit), siempre desunido, desafinado, fracturado, dividido (zwiespältig) por la sexualidad, hacia un sexo determinado (in eine bestimmte Geschlechtlichkeit)". ${ }^{13}$ Contrariamente a lo que podría pensarse,

${ }^{12}$ Ibid., pp. 405-406.

${ }^{13}$ Ibid., p. 406. 
esta dispersión o diseminación (Zerstreuung), y sus variantes, marcadas por el prefijo zer (Zerstreutheit, Zersplitterung, Zerspaltung, entre otras) no es negativa, ("It's de-lovely", podría decir Cole Porter), y no lo es (como también podría afirmar Cole Porter) porque se trata de otra cosa; en el caso de Heidegger, esta otra cosa, escondida en los pliegues del significado, es la que marca el pliegue de la multiplicación, una multiplicación positiva (Mannigfaltigung) que Heidegger se cuida de distinguir de una simple multiplicidad (Mannigfaltigkeit) o diversidad.

Se trata de otra cosa para Heidegger, "Mais il s'agit ici de tout autre chose" traduce Derrida, pero también se trata de otra cosa para Derrida, esta otra cosa es el paso mismo por el que atravesamos del texto de Heidegger al de Derrida, por el que pasamos del zer germano al dis greco-latino; este paso, el de la traducción, conlleva ciertas transferencias y desplazamientos que se agravan en la medida misma en que ambos prefijos pueden indicar tanto sentidos negativos como neutros y, en ciertos casos, como el que surge de la lectura derridiana de Heidegger, ciertamente positivos. Estos pasos y los problemas que conllevan, insiste Derrida, no pueden considerarse accidentales o de poca monta; no pueden considerarse casuales, podemos agregar, porque en cierta medida de ellos depende el desplazamiento de su interpretación. De aquí que, por un lado, Derrida identifique la reflexión acerca del Geschlecht con la reflexión acerca de la traducción y, por otro, se abstenga de declarar como positivos los aspectos neutros o no negativos de los prefijos en cuestión; su duda es la duda que conlleva toda interpretación y, tal vez, toda traducción. En las líneas que preceden a su traducción-interpretación de la Zerstreuung dice:

Es el análisis de este aislamiento [el aislamiento metafísico del hombre] el que hace entonces resurgir el tema de la diferencia sexual y del reparto [partage] dual en la Geschlechtlichkeit. En el centro de este nuevo análisis, la diferenciación muy fina de un cierto léxico ya anuncia los problemas de traducción que no dejarán de agravarse para nosotros. Nunca será posible considerarlos como accidentales o secundarios. En un momento determinado, nos podremos incluso dar cuenta que el pensamiento de la Geschlecht y el pensamiento de la traducción son esencialmente el mismo. Aquí el enjambre léxico reúne (o disgrega) la serie "disociación", "distracción", "diseminación", "división", "dispersión". El dis-, entonces, será lo que se supone traduce, no sin transferencia y desplazamiento, el zer-de la Zerstreuung, Zerstreutheit, Zerstörung, Zersplitterung, Zerspaltung. Pero una frontera interior y suplementaria divide nuevamente este léxico: dis- y zer-tienen quizás 
un sentido negativo, pero a veces también un sentido neutro o no negativo (dudo aquí decir positivo o afirmativo). ${ }^{14}$

La idea, teñida de amenaza, de que "la diferenciación muy fina de un cierto léxico ya anuncia los problemas de traducción que no dejarán de agravarse para nosotros" y la identificación misma entre el pensamiento del Geschlecht y el de la traducción no dejan de ser tanto sorprendentes como sugerentes. Entrando en el juego de los ecos y las resonancias tal vez podríamos relacionar esta identificación derridiana con la noción freudiana de traducción y, en particular, con la de "falla de traducción". En la famosa Carta 52, Freud le explica a Fliess su idea acerca de que el "mecanismo psíquico" es el resultado de un proceso de estratificación, esto es, que el material de ese mecanismo (las memorias) está sujeto a "reacomodos" que ajustan ese material a las circunstancias; este ajuste conlleva entonces una "retranscripción". De esta manera, el paso de una época de la vida a otra, implica una traducción del material psíquico; de aquí que, piensa Freud, las peculiaridades de las llamadas psiconeurosis puedan ser explicadas a través de la suposición de que, en relación con cierto material, esta traducción no ha tenido lugar y que la falta de traducción se relaciona con la naturaleza del material. Cuando esto pasa se genera un anacronismo que sobrevivirá gracias a que, dice Freud, "en una provincia particular prevalecen fueros, esto es, privilegios que excluyen la aplicación de la ley". Consecuentemente, es esta falla en la traducción la que Freud identifica con la represión. El motivo o causa de esta falla en la traducción o represión, piensa Freud, está siempre relacionado con "la liberación de displacer generado por una traducción", es como si, continúa Freud, "este displacer provocara un disturbio en el pensamiento que impide el trabajo de traducción". ${ }^{15}$ Casi veinte años más tarde, en su trabajo "El inconsciente", de 1915, Freud se pregunta cómo podemos llegar a conocer lo inconsciente y, otra vez echando mano de la noción de traducción, responde: "Desde luego es sólo como algo consciente que lo conocemos, después de que ha pasado por una transformación o traducción en algo consciente". ${ }^{16}$ En este sentido, la identificación que sugiere Derrida podría estar orientada en la misma dirección que la idea freudiana de que nuestro saber del inconsciente y la sexualidad conlleva una traducción, una represión y ciertos "fueros". Por otro lado, tam-

${ }^{14}$ Ibid., p. 405.

15 Jeffrey Moussaieff Masson, ed., The Complete Letters of Sigmund Freud to Wilhem Fliess, 1887-1904. Trad. de J. Moussaieff Masson. Cambridge, Universidad de Harvard, 1985, p. 208.

${ }^{16}$ Sigmund Freud, "The Unconscious", en The Standard Edition of the Complete Psychological Works of Sigmund Freud. Londres, The Hogarth Press, 1981, p. 166. 
bién podría apuntar a ciertas áreas de la interpretación de Heidegger donde tal vez sobrevivan ciertos "fueros".

Tal vez fuera entonces prudente preguntarnos: ¿qué clase de traducción está en juego cuando tratamos de pasar del lenguaje del Dasein al lenguaje de los hombres, al lenguaje del Dasein encarnado?, ¿̇e generará allí también una falla de traducción, una falla que pueda hacer manifiesto un "disturbio en el pensamiento", o ¿se tratará acaso de una Aufgabe (tarea, renuncia) benjaminiana, imposible, tanto de llevar a cabo como de abandonarla? En cualquier caso, la interpretación del silencio de Heidegger con respecto a la sexualidad conlleva problemas de traducción que implican desplazamientos lo suficientemente radicales como para transferir el peso de la neutralidad del ámbito de la positividad al de la negatividad y, con ello, abrir la posibilidad de darle un lugar a la sexualidad en el pensamiento ontológico. Si, como ya vimos, la neutralidad puede ser considerada como un efecto de la negatividad asociada con la sexualidad dual, entonces podemos extraerla del ámbito del Dasein y, con ello, hacer patente que el Dasein, lejos de ser neutral, está imbuido de una positividad que queda oscurecida por los afanes de neutralidad ontológica. Estos resultados no son antitéticos con el espíritu de la filosofía heideggeriana, ya que, como nos lo recuerda Derrida, para Heidegger es fundamental la positividad originaria del Dasein.

Sin embargo, también es claro que el paso del zer-, germánico y heideggeriano, al dis-, latino y derridiano, conlleva una diseminación que parece consustancial tanto a la traducción como a la sexualidad misma. Volviendo a Cole Porter, ¿cómo traducir "de-lovely", especialmente cuando notamos el lugar que ocupa en la serie: "It's delightful, is delicious, is delectable, is dilemma, is delirious, is... de-lovely?", ¿cómo culmina algo que es delicioso, deleitoso, deleitable, dilema, delirante? ¿Debemos interpretar el prefijo como enfatizando lo adorable, lo encantador, o como marcando, vía el delirio y el dilema, la pérdida de esas cualidades? Si bien es cierto que la forma más popular de escuchar la línea en cuestión la asocia con cierta exaltación amorosa, también es cierto que ese extremo (el amoroso y el de la línea) puede denotar cierta irritación, cierta exacerbación y, con ella, una lectura muy distinta. Dados los retorcimientos a los que el autor somete el lenguaje, para no mencionar los personales, no es difícil relacionar esa exacerbación amorosa con la privación, separación y negación que, gramaticalmente, se indican por el uso del prefijo "de"; siguiendo este camino "de-lovely" debería interpretarse como el destino de un proceso que empieza siendo delicioso, pasa por el delirio y culmina con el desamor.

En resumen, la lectura atenta del silencio heideggeriano con respecto a la sexualidad le permite a Derrida convertir a la negatividad, atribuida a la sexualidad binaria, en la fuente misma de la neutralización, exponiendo así 
la violencia que esa neutralización ejerce sobre la naturaleza del Dasein; la violencia que se podría atribuir a su lectura es reconocida como tal pero se le regresa a su origen: el texto mismo. De esta manera, si Derrida tiene razón en pensar que la neutralidad, con la que se caracteriza y protege al Dasein, es un efecto de la negatividad atribuida a la sexualidad, entendida como esencialmente diádica, entonces podemos desplazar esa neutralidad y devolverle al Dasein su positividad originaria. Este cuestionamiento de la neutralidad como una característica positiva del Dasein se refuerza cuando recordamos que él, el Dasein, conlleva o abriga en sí, la posibilidad de una dispersión, de una diseminación factual. Si insistimos, con Derrida, que es el cuerpo propio mismo, la carne, la que lleva a la dispersión y a la diferenciación sexual, entonces la neutralidad atribuida tanto al Dasein como a la Selbsheit generaría cierta extrañeza, pues contrastaría con la positividad fundamental de ambas nociones. De esta manera, en el momento en que desplazamos a la neutralidad fuera del aura positiva propia del Dasein y le abrimos a éste la posibilidad de apropiarse de un cuerpo que, por esa misma apropiación, estará sexuado, el cuerpo y la sexualidad misma quedarán marcados por la dispersión y la diseminación. Esta dispersión, casi por definición, no será diádica y, al no serlo, le abre las puertas a una reflexión acerca de la corporalidad y la sexualidad que no esté constreñida y delimitada por las oposiciones binarias. Las complejidades y ramificaciones de este resultado son múltiples y se encuentran tan diseminadas como el Dasein mismo, pero, podemos pensar, no le serían ajenas a Cole Porter, quien, tal vez, podría insistir: "It's delightful, is delicious, is delectable, is dilemma, is delirious, is... de-lovely". 